\title{
MR flow measurement of coronary artery bypass graft and stress myocardial perfusion MRI for the detection of graft stenosis
}

Tatsuro Ito ${ }^{*}$, Masaki Ishida ${ }^{1}$, Kakuya Kitagawa ${ }^{1}$, Hiroshi Nakajima² ${ }^{2}$ Kaoru Dohi ${ }^{2}$, Shinji Kanemitsu ${ }^{3}$, Hideto Shimpo ${ }^{3}$, Masaaki Ito' ${ }^{2}$, Hajime Sakuma'

From 16th Annual SCMR Scientific Sessions

San Francisco, CA, USA. 31 January - 3 February 2013

\section{Background}

Stress myocardial perfusion MRI allows for accurate detection of flow-limiting stenosis in the coronary artery. However, reduced diagnostic accuracy of stress myocardial perfusion MRI was reported in patients with coronary artery bypass grafts (CABG). Flow measurement in $C A B G$ is another MR approach that permits functional assessment of graft stenosis. The purpose of this study was to evaluate the value of MR study that combines MR flow measurement of CABG and stress myocardial perfusion MRI in detecting graft stenosis.

\section{Methods}

Thirty-two consecutive patients ( 26 men, $69.0 \pm 7.0$ years) with previous $C A B G$ surgery who had recurrent chest pain were studied. All patients gave informed consent and underwent both coronary angiography and cardiac MRI. After obtaining cine MRI, stress-rest myocardial perfusion MRI and late gadolinium enhanced (LGE) MRI, blood flow volume in the resting state was quantified in 61 graft conduits by using phase contrast cine MRI. The presence or absence of myocardial ischemia was visually determined by identifying stress-induced hypoenhancement in the absence of LGE or hypoenhancement larger than LGE. Stenoses $\geq 70 \%$ in grafts or grafted native vessels were considered significant on coronary angiography.

\section{Results}

Coronary angiography revealed significant stenoses in the grafts or grafted native vessels in 19 (31\%) of the 61 CABG conduits. Stress-rest perfusion CMR alone yielded the sensitivity and specificity of $73 \%$ and $81 \%$, respectively. Receiver operating characteristic analysis demonstrated that the optimal cutoff values of MR blood flow volume was $28 \mathrm{~mL} / \mathrm{min}$ for predicting significant stenosis on angiography . By combining MR blood flow measurement and stress-rest perfusion MRI, the sensitivity and specificity of MR study were improved to $95 \%$ and $81 \%$.

\section{Conclusions}

MR flow measurement of CABG combined with stressrest perfusion MRI can provide excellent diagnostic accuracy for predicting significant stenoses in the grafts or grafted native vessels in patients with previous CABG.

\section{Funding}

Departmental reseach funding.

\section{Author details}

'Department of Radiology, Mie University Hospital, Tsu-shi, Mie, Japan. ${ }^{2}$ Department of Cardiology, Mie University Hospital, Tsu-shi, Mie, Japan. ${ }^{3}$ Department of Thoracic and Cardiovascular surgery, Mie University Hospital, Tsu-shi, Mie, Japan.

Published: 30 January 2013

\section{doi:10.1186/1532-429X-15-S1-P178}

Cite this article as: Ito et al: MR flow measurement of coronary artery bypass graft and stress myocardial perfusion MRI for the detection of graft stenosis. Journal of Cardiovascular Magnetic Resonance 2013 15(Suppl 1):P178.

'Department of Radiology, Mie University Hospital, Tsu-shi, Mie, Japan

Full list of author information is available at the end of the article

(C) 2013 Ito et al; licensee BioMed Central Ltd. This is an Open Access article distributed under the terms of the Creative Commons Attribution License (http://creativecommons.org/licenses/by/2.0), which permits unrestricted use, distribution, and reproduction in any medium, provided the original work is properly cited. 